\title{
Dynamics and Stability of Hopf Bifurcation for One Non-linear System
}

\author{
Vahidin Hadžiabdić, Midhat Mehuljić, Jasmin Bektešević, Adnan Mašić \\ Faculty of Mechanical Engineering, Department of Mathematics and Physics / University of Sarajevo, \\ Sarajevo, Bosnia and Herzegovina
}

\begin{abstract}
In this paper we will look at the one system of ODE and analyze it. We aim to determine the points of equilibrium; examine their character and establish the existence of a bifurcation for the corresponding parameter value. A detailed analysis of local stability was performed for all values of the given parameter. For a certain value of the parameter, the existence of supercritical Hopf bifurcation of the observed system of differential equations has been proved. Also, the existence of a limit cycle that is always stable has been proved.
\end{abstract}

Keywords - Equilibrium Point, Stability, Nullcline, Bifurcation.

\section{Introduction}

We will study the system given in the form

$$
\left\{\begin{array}{l}
\dot{x}=f_{\mu}(x, y), \\
\dot{y}=g_{\mu}(x, y) .
\end{array}\right.
$$

Hopf bifurcation occurs when a periodic solution or boundary cycle, which surrounds the equilibrium point, appears or disappears with a change in parameter value.

\section{DOI: 10.18421/TEM102-40}

https://doi.org/10.18421/TEM102-40

Corresponding author: Vahidin Hadžiabdić,

Faculty of Mechanical Engineering, Department of Mathematics and Physics / University of Sarajevo, Sarajevo, Bosnia and Herzegovina.

Email: hadziabdic@mef.unsa.ba

Received: 10 September 2020.

Revised: 29 January 2021.

Accepted: 08 February 2021.

Published: 27 May 2021.

(cc)BY-NC-ND (C) 2021 Vahidin Hadžiabdić et al; published by UIKTEN. This work is licensed under the Creative Commons Attribution-NonCommercial-NoDerivs 4.0 License.

The article is published with Open Access at www.temjournal.com
The equilibrium point can in many ways lose stability when changing a parameter.

The type of bifurcation can be determined analytically, but it is very complicated. However, using computers (numerical experiments), we determine the type of bifurcation much faster and easier.

In the case where a stable limit cycle surrounds an unstable equilibrium point, Hopf's bifurcation is called supercritical, and when an unstable limit cycle surrounds a stable equilibrium point, Hopf's bifurcation is called subcritical. Supercritical and subcritical Hopf bifurcations were observed in [1].

We have much better insight into the behavior of Hopf bifurcation systems if we know the stability of the limit cycle, the amplitude, and the period of the periodic trajectory.

The amplitude of the periodic trajectory behaves as $\sqrt{|\mu|}$, which is actually an approximation of the true value of the amplitude. In the case of differential equations with delay, the amplitude of the limit cycle can be analytically determined much more precisely [2].

Hopf bifurcation is also known as Poincare Andronov - Hopf bifurcation. The very notion of Hopf bifurcation sounds complicated and abstract. However, this theory is applied to many real problems.

It has its application in tests of various oscillations caused by wind gusts (which is of great importance in the field of construction), LCR oscillations in electric circuits, periodic creation of nerve impulses in the nervous system, as well as, for example, in epidemiological models which describe fluctuations in the number of patients with an infectious disease.

We conclude that the range of application of this theory is quite broad.

B. Sang observed Hopf bifurcation with the Genesio-Tesi system [3]. F.Wu and Y.Jiao have observed Hopf bifurcation in predator-prey model [4].

Hopf bifurcation occurs in many epidemiological models, both in normal and delayed ones. An analysis of one such model, using method of multiple scale, was observed in [5]. 


\section{A review of Some Literature}

The first works on this topic can be found in Ebergard Hopf: Abzweigung einer periodischen Lösung von einer stationären Lösung eines Differentialsystems, Berichten der MathematischPhysischen Klasse der Sächsischen Akademie der Wissenschaften zu Leipzig XCIV, 1-22 (1942) [15]. The English translation of this work is given in The Hopf Bifurcation and Its Applications [16]. Following the time period the following works appeared: A mathematical example displaying features of turbulence [17], On some cases of periodic motions depending on parameters [18], On invariant surfaces and bifurcations of periodic solutions of ordinary differential equations [19], Theory of Oscillators [20], The Hamiltonian Hopf Bifurcation [21]. In addition to these scientific papers, which started and founded this theory, so far there are a very large number of papers and applications in various fields, such as [3], [4], [14], [22].

\section{Dynamics of System}

In this work we will considers the following system of ODE

$$
\left\{\begin{array}{c}
\dot{x}=y(1+x)+\mu x+(\mu-1) x^{2} \\
\dot{y}=-x(1-x)
\end{array}\right.
$$

For this model we will establish equilibrium points, and their character.

Also, we will determinate values of parameter $\mu$ when system (1) have bifurcation.

Lemma 1: System (2) have always two different equilibrium points $\mathrm{A}(0,0)$ and $\mathrm{B}\left(1, \frac{1}{2}(1-2 \mu)\right)$.

Proof: Equilibrium points are solutions of equations

$$
\begin{gathered}
y(1+x)+\mu x+(\mu-1) x^{2}=0 \\
-x(1+x)=0 .
\end{gathered}
$$

By direct calculation we get points A and B. System (1) can be written in matrix form as

$$
\dot{\boldsymbol{x}}=\left(\begin{array}{c}
y(1+x)+\mu x+(\mu-1) x^{2} \\
-x(1-x)
\end{array}\right) .
$$

The Jacobian matrix of this map is given by

$$
D_{f}=\left(\begin{array}{cc}
\mu+2(-1+\mu) x+y & 1+x \\
-1+2 x & 0
\end{array}\right) .
$$

For point $\mathrm{A}(0,0)$ we have the Jacobina matrix

$$
D_{f}(A)=\left(\begin{array}{cc}
\mu & 1 \\
-1 & 0
\end{array}\right) .
$$

Determinant and trace of this matrix is

$$
\operatorname{det}\left(D_{f}(A)\right)=1 \text {, and } \operatorname{tr}\left(D_{f}(A)\right)=\mu \text {. }
$$

Since $\operatorname{det}\left(D_{f}(A)\right)=1>0$, we will find

$$
\left(\operatorname{tr}\left(D_{f}(A)\right)\right)^{2}-4 \operatorname{det}\left(D_{f}(A)\right)=\mu^{2}-4 .
$$

Now we can see, that if

$$
\mu^{2}-4 \geq 0 \Leftrightarrow \mu \in[-\infty,-2] \cup[2,+\infty],
$$

Equilibrium point $\mathrm{A}(0,0)$ is node, furthermore, it is stable for $\mu \in(-\infty,-2]$ and unstable for $\mu \in[2, \infty)$. If $\mu \in(-2,2)$ and $\mu \neq 0$ we have that ponit $\mathrm{A}(0,0)$ is focus, moreover it is stable for $\mu \in(-2,0)$ and unstable for $\mu \in(0,2)$. For $\mu=0$ equilibrium point $\mathrm{A}(0,0)$ is center.

The above consideration can be summarized in the following theorem [6].

Theorem 1: The equilibrium point $\mathrm{A}(0,0)$ is:

a) real sink for $\mu \in(-\infty,-2]$,

b) spiral sink for $\mu \in(-2,0)$,

c) center if $\mu=0$,

d) spiral source for $\mu \in(0,2)$,

e) real source for $\mu \in[2, \infty)$.

We can also explore character of equilibrium point $\mathrm{B}\left(1, \frac{1}{2}(1-2 \mu)\right)$. For point $\mathrm{B}\left(1, \frac{1}{2}(1-2 \mu)\right)$ we have the Jacobina matrix

$$
D_{f}(B)=\left(\begin{array}{cc}
\frac{(4 \mu-3)}{2} & 2 \\
1 & 0
\end{array}\right) .
$$

Determinant of $D_{f}(B)$ is $\operatorname{det}\left(D_{f}(B)\right)=-2<0$. Eigenvalues are of different signs and the equilibrium point $\mathrm{B}\left(1, \frac{1}{2}(1-2 \mu)\right)$ is saddle. Therefore, we have the following statement [7], [8]:

Theorem 2: The equilibrium point $\mathrm{B}\left(1, \frac{1}{2}(1-2 \mu)\right)$ is saddle point.

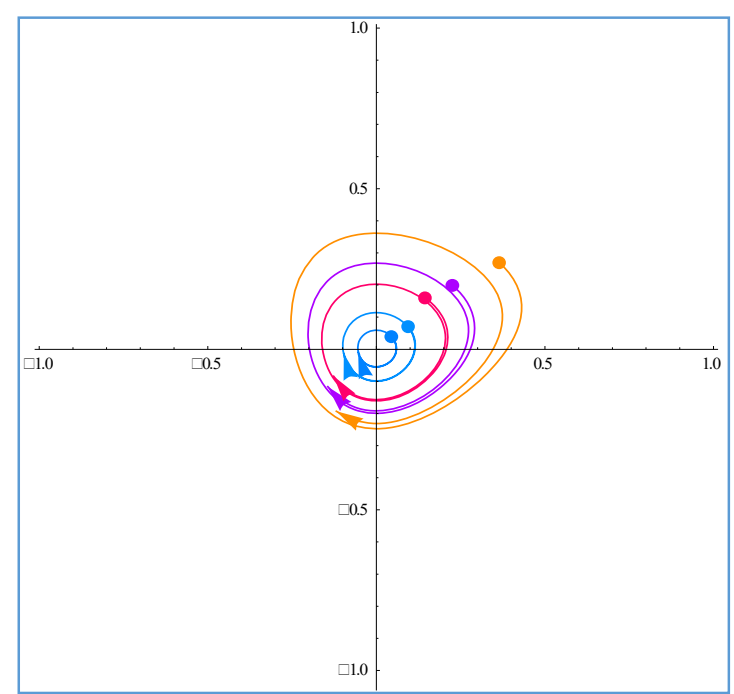

Figure 1. Behavior of the solution for $\mu=0$. 


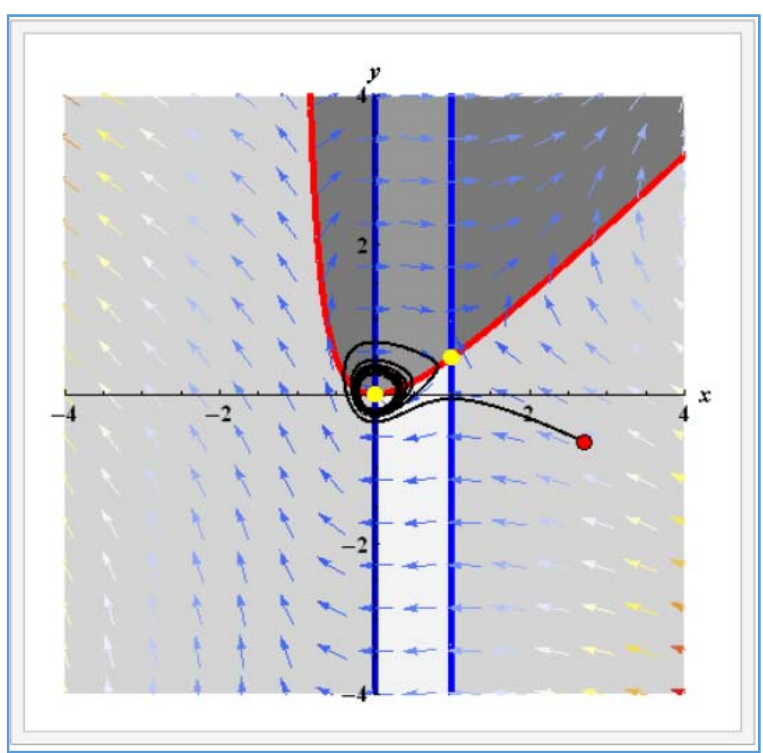

Figure 2. The nullclines of the solution for $\mu=0$

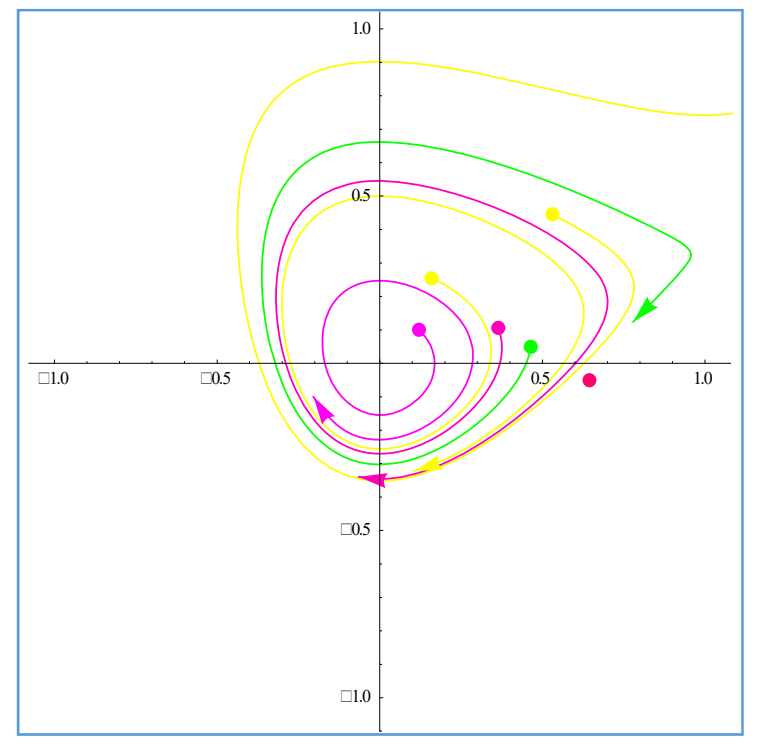

Figure 3. Behavior of the solution for $\mu=0.14$

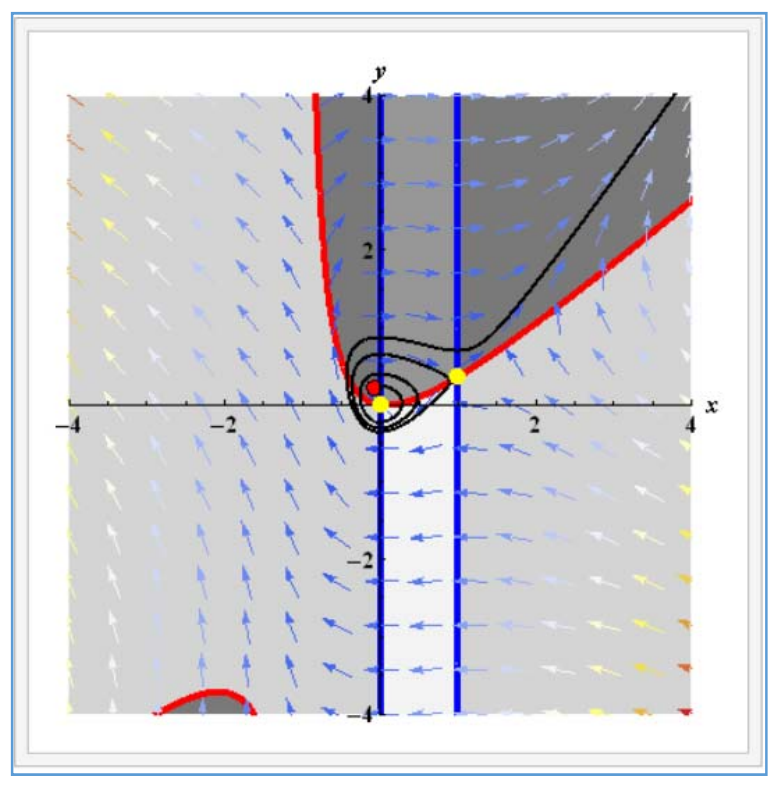

Figure 4. The nullclines of the solution for $\mu=0.14$
We can see that our system (2) has rich dynamics, and that the character of point $\mathrm{A}(0,0)$ changes as we stated in the Theorem 1, depending on the parameter $\mu$.

From Figures 1 and 2 we see that depending on the starting point, the solutions of our system will tend to the solutions of the system (2) will tend to either point $\mathrm{A}(0,0)$. Looking at Figures 3 and 4 we see that the solutions will tend towards infinity $(-\infty,+\infty)$ or $(+\infty,+\infty)$. From the fact that the point character changes, we will examine if we have Hopf bifurcation for some parameter value $\mu$ or this purpose we will use the theory developed in [9],[10].

If we compute eigenvalues of $D_{f}(A)$, we get

$$
\lambda_{1,2}=\frac{\left(\mu \pm \sqrt{\mu^{2}-4}\right)}{2} .
$$

We can see that if $\mu^{2}-4<0$, the corresponding eigenvalues will be conjugated complex. Let the corresponding eigenvalues be given in the form

$$
\lambda_{1,2}=\alpha(\mu) \pm \beta(\mu) i
$$

We can consider the situation when $\mu \in(-2,2)$, that is, when we have conjugated complex eigenvalues.

It is possible to check if the following conditions are satisfied in Hopf's bifurcation theorem for the value of $\mu=0$ [11], [12], [13]:

1. $\alpha(0)=0, \beta(0)=\omega \neq 0$,

2. $\operatorname{sgn}(\omega)=\operatorname{sgn} \frac{\partial g_{\mu}}{\partial x}(0,0)$ for $\mu=0$,

3. $\frac{\partial \alpha(\mu)}{\partial \mu}=d \neq 0$, for $\mu=0$,

4. $a=\frac{1}{16}\left[\frac{\partial^{3} f_{\mu}}{\partial x^{3}}(0,0)+\frac{\partial^{3} f_{\mu}}{\partial x \partial y^{2}}(0,0)+\frac{\partial^{3} g_{\mu}}{\partial y^{3}}(0,0)\right]+$ $\frac{1}{16 \omega}\left[\frac{\partial^{2} f_{\mu}}{\partial x \partial y}(0,0)\left(\frac{\partial^{2} f_{\mu}}{\partial x^{2}}(0,0)+\frac{\partial^{2} f_{\mu}}{\partial y^{2}}(0,0)\right)-\right.$ $\frac{\partial^{2} g_{\mu}}{\partial x \partial y}(0,0)\left(\frac{\partial^{2} g_{\mu}}{\partial x^{2}}(0,0)+\frac{\partial^{2} g_{\mu}}{\partial y^{2}}(0,0)\right)-$ $\left.\frac{\partial^{2} f_{\mu}}{\partial x^{2}}(0,0) \frac{\partial^{2} g_{\mu}}{\partial x^{2}}(0,0)+\frac{\partial^{2} f_{\mu}}{\partial y^{2}}(0,0) \frac{\partial^{2} g_{\mu}}{\partial y^{2}}(0,0)\right]$ for $\mu=0$,

5. $a \neq 0$.

Poincaré had his first suggestions about this theorem in a two-dimensional case around 1890 , until concrete formulation and evidence came from Andronov and his associates circa 1930. In 1942, Hopf proved this theorem for the system finite dimensions.

For our system (2) and point $\mathrm{A}(0,0)$ we have

$$
\alpha(\mu)=\frac{\mu}{2}, \beta(\mu)=\frac{\sqrt{4-\mu^{2}}}{2} .
$$

By direct calculation we have that 


$$
\begin{gathered}
d=\frac{1}{2}, \quad \beta(0)=1, \\
\frac{\partial g_{\mu}}{\partial x}(0,0)=-1,
\end{gathered}
$$

so it's effective $\omega=-1$ and

$$
a=\frac{-\mu+1}{8 \omega}=\frac{\mu-1}{8} \text {. }
$$

We see that $a \neq 0$ if $\mu \neq 1$. $a d<0$ for $\mu<1$ and $a d>0$ for when $\mu>1$, and the boundary cycle occurs for given the specified parameter $\mu$ values. So we conclude that all conditions for the existence of Hopf bifurcation are fulfilled.

Theorem 3: If $0<\mu<1$, then

a) there is a limit cycle which is always stable,

b) Hopf bifurcation is supercritical.

Proof:

a) By Hopf bifurcation theorem the limit cycle occurs in cases where:

- $\mu>0$ if $a d<0$

- $\mu<0$ if $a d>0$.

Since $a d>0$ for $\mu>1$ the second case is not possible. It remains that it is $a d<0$ only for $0<\mu<1$.

We have that $d=\frac{1}{2}>0$ and therefore the equilibrium point $A(0,0)$ is unstable [9]. Since the equilibrium point is unstable for given values, the limit cycle is stable [9].

b) Since in this case we have a stable limit cycle surrounding the unstable equilibrium point, by definition, we have supercritical Hopf bifurcation.

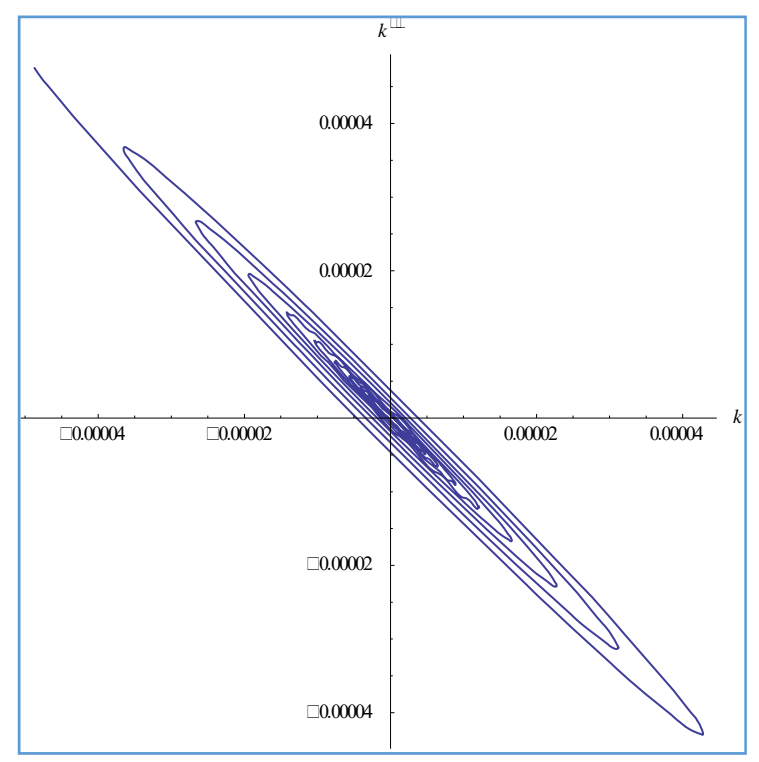

Figure 5. Occurrence of bifurcation for $\mu=-0.1$

In Figure 5 we can see the limit cycles for a given value of the parameter $\mu$.

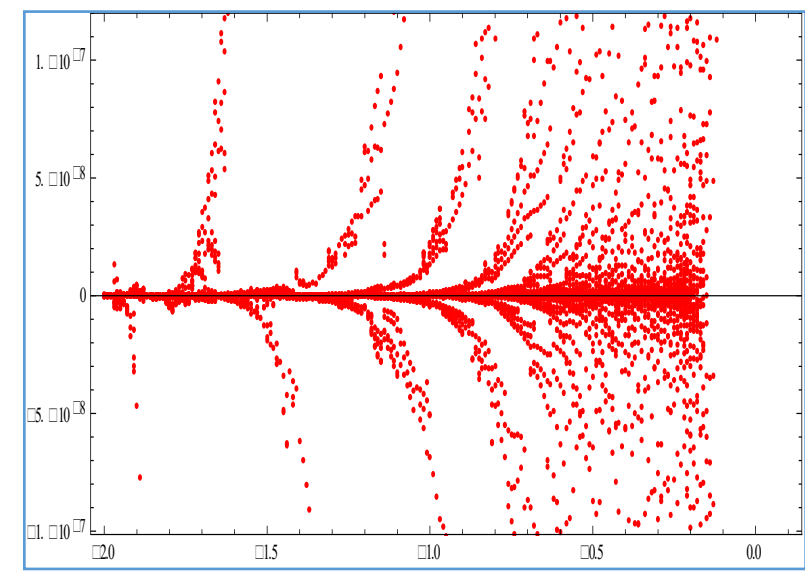

Figure 6. Bifurcation diagram

One way we can show the behavior of a dynamic system is via a bifurcation diagram in Figure 6. In the bifurcation diagram, we observe the relationship between the value of a parameter of an external excitation and the system response to that excitation.

\section{Conclusion}

Observation of Hopf bifurcation in multidimensional dynamical systems which depends on some parameter is not an easy task. Many contemporary scholars are still struggling to find the best and software best suited ways to get it solutions made, that is, to approximate the parameter corresponding to Hopf's point.

In this study, we addressed the problem of stability of multidimensional one-parameter dynamic systems caused by the appearance of Hopf bifurcation. We have seen how this problem is formulated mathematically and what tools are used to solve it. We have been doing this whole process with the aim of identifying all the unknowns that occur in the occurrence of Hopf's bifurcation. The behavior of the system for some parameter values and the bifurcation diagram are given in the Figures: $1,2,3,4,5$ and 6 . What could be explored in the future is the generalization of ODE systems themselves, when instead of some concrete expressions, we would consider functional expressions. Hopf bifurcation can also occur in BAM neural networks. BAM neural networks have practical application in storing paired patterns and have the ability to search for desired patterns in both directions, forward and backward. A BAM (Bidirectional Associative Memory) network can store multiple samples unlike other types of neural networks which have only one sample container or one memory sample. Global network stability is affected by a stable equilibrium point of the system that represents a single pattern for storage or sample memory. 


\section{References}

[1]. Yuan, C., \& Wang, J. (2016). Hopf bifurcation analysis and control of three-dimensional Prescott neuron model. Journal of Vibroengineering, 18(6), 4105-4115. doi:10.21595/jve.2016.16933.

[2]. Molnár, T., Insperger, T., \& Stépán, G. (2016). Analytical estimations of limit cycle amplitude for delay-differential equations. Electronic Journal of Qualitative Theory of Differential Equations, 2016(77), 1-10. doi: $10.14232 /$ ejqtde.2016.1.77

[3]. SANG, B. (2019). Hopf Bifurcation Formulae And Applications To The Genesio-Tesi System. Journal of Nonlinear Functional Analysis, 2019, 34. DOI: $10.23952 /$ jnfa.2019.34

[4]. Wu, F., \& Jiao, Y. (2019). Stability and Hopf bifurcation of a predator-prey model. Boundary Value Problems, 2019(1), 1-11.

[5]. Wang, W., \& Chen, L. (2016). Stability and Hopf Bifurcation Analysis of an Epidemic Model by Using the Method of Multiple Scales. Mathematical Problems in Engineering, 2016, 1-8. https://doi.org/10.1155/2016/2034136

[6]. Hirsch, M. W., Smale, S., \& Devaney, R. L. (2012). Differential equations, dynamical systems, and an introduction to chaos. Academic press.

[7]. Perko, L. (2013). Differential equations and dynamical systems (Vol. 7). Springer Science \& Business Media.

[8]. Verhulst, F. (1996). Nonlinear differential equations and dynamical systems. 2nd ed. Springer-Verlag Berlin Heidelberg.

[9]. Strogatz, S.H. Nonlinear Dynamics and Chaos: With Applications to Physics, Biology, Chemistry and Engineering. $1^{\circ}$ Edição. Westview Press, 2001, p.512.

[10]. Bernard, O., \& Gouzé, J. L. (2002). Global qualitative description of a class of nonlinear dynamical systems. Artificial Intelligence, 136(1), 2959.

https://doi.org/10.1016/S0004-3702(01)00169-2
[11]. Guerrini, L. (2017). Hopf Bifurcation Analysis of a Dynamical Heart Model with Time Delay. Applied Mathematical Sciences, 11(22), 1089-1095. https://doi.org/10.12988/ams.2017.

[12]. Kuznetsov, Yu.A., (2004)., Elements of Applied Bifurcation Theory (3rd edn) (New York: Springer), doi.org/10.1007/978-1-4757-3978-7.

[13]. Scott, A. (Ed.). (2005). Encyclopedia of Nonlinear Science (1st ed.). Routledge. https://doi.org/10.4324/9780203647417

[14]. Cao, J., \& Xiao, M. (2007). Stability and Hopf bifurcation in a simplified BAM neural network with two time delays. IEEE Transactions on Neural Networks, 18(2), 416-430.

[15]. Hopf, E. (1942). Abzweigung einer periodischen Lösung von einer stationären Lösung eines Differentialsystems. Ber. Math.-Phys. Kl Sächs. Akad. Wiss. Leipzig, 94, 1-22.

[16]. Marsden, J. E., \& McCracken, M. (2012). The Hopf bifurcation and its applications (Vol. 19). Springer Science \& Business Media.

[17]. Hopf, E. (1948). A mathematical example displaying features of turbulence. Communications on Pure and Applied Mathematics, 1(4), 303-322.

[18]. Neimark, J. (1959). On some cases of periodic motions depending on parameters. In Dokl. Akad. Nauk SSSR (Vol. 129, pp. 736-739).

[19]. Sacker, R. J. (1965). A new approach to the perturbation theory of invariant surfaces. Communications on Pure and Applied Mathematics, 18(4), 717-732.

[20]. Andronov, A. A., Vitt, A. A., \& Khaikin, S. E. (1966). Theory of oscillators. Translated from the Russian by F. Immirzi; translation edited and abridged by W.

[21]. Van Der Meer, J. C. (1985). The hamiltonian Hopf bifurcation. The Hamiltonian Hopf Bifurcation, 66-83. https://doi.org/10.1007/BFb0080362

[22]. Zhang, H., Kang, J., Huang, T., Cong, X., Ma, S., \& Huang, H. (2018). Hopf Bifurcation, Hopf-Hopf Bifurcation, and Period-Doubling Bifurcation in a Four-Species Food Web. Mathematical Problems in Engineering, 2018, 1-21. https://doi.org/10.1155/2018/8394651 